\title{
The comparison between nuclear ribosomal DNA and chloroplast DNA in molecular systematic study of four sections of genus Dendrobium sw. (Orchidaceae)
}

\begin{abstract}
Phylogenetic study of the four sections (Aporum, Crumenata, Strongyle, and Bolbidium) of genus Dendrobium (family Orchidaceae) was conducted using molecular data. Classifications based on morphological characters have not being able to clearly divide these four sections neither do they supported their monophyly origin. Therefore, deeper and detailed analysis especially using molecular data is required to ascertain their status. Molecular evidences were used to clarify their relations either to lump them into one section or reduce them into two. The study has been carried out for the 34 species of Dendrobium using Maximum Parsimony (MP). Three nucleotide sequences data sets from two distinct genomes chloroplast DNA genes (rbcL and matK) and nuclear ribosomal DNA (ITS) were used to construct cladograms. The results that obtained from the Internal Transcribed Spacer (ITS) gene showed that the nuclear genes are reliable marker for the phylogenetic study of Dendrobium compared to chloroplast DNA with low resolution level among sections.
\end{abstract}

Keyword: Dendrobium; Phylogeny; ITS; rbcL; matK 